\title{
Stress on the seismogenic and deep creep plate interface during the earthquake cycle in subduction zones
}

\author{
Larry J. Ruff \\ Department of Geological Sciences, University of Michigan, Ann Arbor, MI 48109, U.S.A.
}

(Received September 1, 1999; Revised November 9, 2000; Accepted November 15, 2000)

\begin{abstract}
The deep creep plate interface extends from the down-dip edge of the seismogenic zone down to the base of the overlying lithosphere in subduction zones. Seismogenic/deep creep zone interaction during the earthquake cycle produces spatial and temporal variations in strains within the surrounding elastic material. Strain observations in the Nankai subduction zone show distinct deformation styles in the co-seismic, post-seismic, and inter-seismic phases associated with the 1946 great earthquake. The most widely used kinematic model to match geodetic observations has been a 2-D Savage-type model where a plate interface is placed in an elastic half-space and co-seismic slip occurs in the upper seismogenic portion of the interface, while inter-seismic deformation is modeled by a locked seismogenic zone and a constant slip velocity across the deep creep interface. Here, I use the simplest possible 2-D mechanical model with just two blocks to study the stress interaction between the seismogenic and deep creep zones. The seismogenic zone behaves as a stick-slip interface where co-seismic slip or stress drop constrain the model. A linear constitutive law for the deep creep zone connects the shear stress $(\sigma)$ to the slip velocity across the plate interface $\left(s^{\prime}\right)$ with the material property of interface viscosity $(\zeta)$ as: $\sigma=\zeta s^{\prime}$. The analytic solution for the steady-state two-block model produces simple formulas that connect some spatially-averaged geodetic observations to model quantities. Aside from the basic subduction zone geometry, the key observed parameter is $\tau$, the characteristic time of the rapid post-seismic slip in the deep creep interface. Observations of $\tau$ range from about 5 years (Nankai and Alaska) to 15 years (Chile). The simple model uses these values for $\tau$ to produce estimates for $\zeta$ that range from $8.4 \times 10^{13} \mathrm{~Pa} / \mathrm{m} / \mathrm{s}$ (in Nankai) to $6.5 \times 10^{14} \mathrm{~Pa} / \mathrm{m} / \mathrm{s}$ (in Chile). Then, the model predicts that the shear stress acting on deep creep interface averaged over the earthquake cycle ranges from 0.1 MPa (Nankai) to 1.7 MPa (Chile). These absolute stress values for the deep creep zone are slightly smaller than the great earthquake stress drops. Since the great earthquake recurrence time $\left(T_{\text {recur }}\right)$ is much larger than $\tau$ for Nankai, Alaska, and Chile, the model predicts that rapid post-seismic creep should re-load the seismogenic zone to about (1/3) of the co-seismic change; geodetically observed values range from about (1/10) to more than $(1 / 2)$. Also, for the case of $\left(T_{\text {recur }} / \tau\right) \gg 1$, the model predicts that the slip velocity across the deep creep interface during the inter-seismic phase should be about $(2 / 3)$ the plate tectonic velocity $(R)$. Thus the deep creep velocity used in Savage-type models should be less than $R$. Even complex 3-D models with non-linear creep laws should make a similar prediction for inter-seismic deep creep rates. At present, it seems that geodetic observations at Nankai and other subduction zones are more consistent with a deep creep rate of $R$ rather than $(2 / 3) R$. This discrepancy is quite puzzling and is difficult to explain in the context of a 2-D steady-state earthquake cycle model. Future observational and modeling studies should examine this apparent discrepancy to gain more understanding of the earthquake cycle in subduction zones.
\end{abstract}

\section{Introduction}

Subduction zone plate boundaries are divided into the shallow seismogenic and the deeper creep zones. For those subduction zones that generate large underthrusting earthquakes, the down-dip edge of the seismogenic zone ranges in depth from 25 to $50 \mathrm{~km}$ (Tichelaar and Ruff, 1993). The seismogenic portion of the plate boundary is characterized by stickslip behavior as the plate interface is locked during most of the earthquake cycle. In detail, some parts of the seismogenic plate interface may slip aseismically. The largest sub-regions of the seismogenic plate boundary that are locked during the earthquake cycle are the asperities and they control the recurrence time and size of the largest earthquakes. The deeper

Copy right $(\mathcal{C})$ The Society of Geomagnetism and Earth, Planetary and Space Sciences (SGEPSS); The Seismological Society of Japan; The Volcanological Society of Japan; The Geodetic Society of Japan; The Japanese Society for Planetary Sciences. portion of the plate boundary-from the seismogenic zone edge down to the base of the overlying lithosphere-is aseismic. While there are usually plentiful intra-plate earthquakes in the Wadati-Benioff zone just below the plate interface, there are no under-thrusting earthquakes located on the plate interface in this region, hence plate boundary slip occurs as creep. We do not know the creep law that governs plate slip in this portion. Candidate laws vary from the simplest linear creep law to arbitrarily complicated ones that are capable of producing creep events from a smooth loading history. The rate-and-state-dependent friction law is one example of an interface constitutive law that can produce creep events (e.g., Dieterich, 1981; Rice and Tse, 1986). Several investigators have applied this complicated law to subduction zones to examine the loading of the seismogenic zone by aseismic slip in the deep creep region (e.g., Stuart, 1988). This friction 


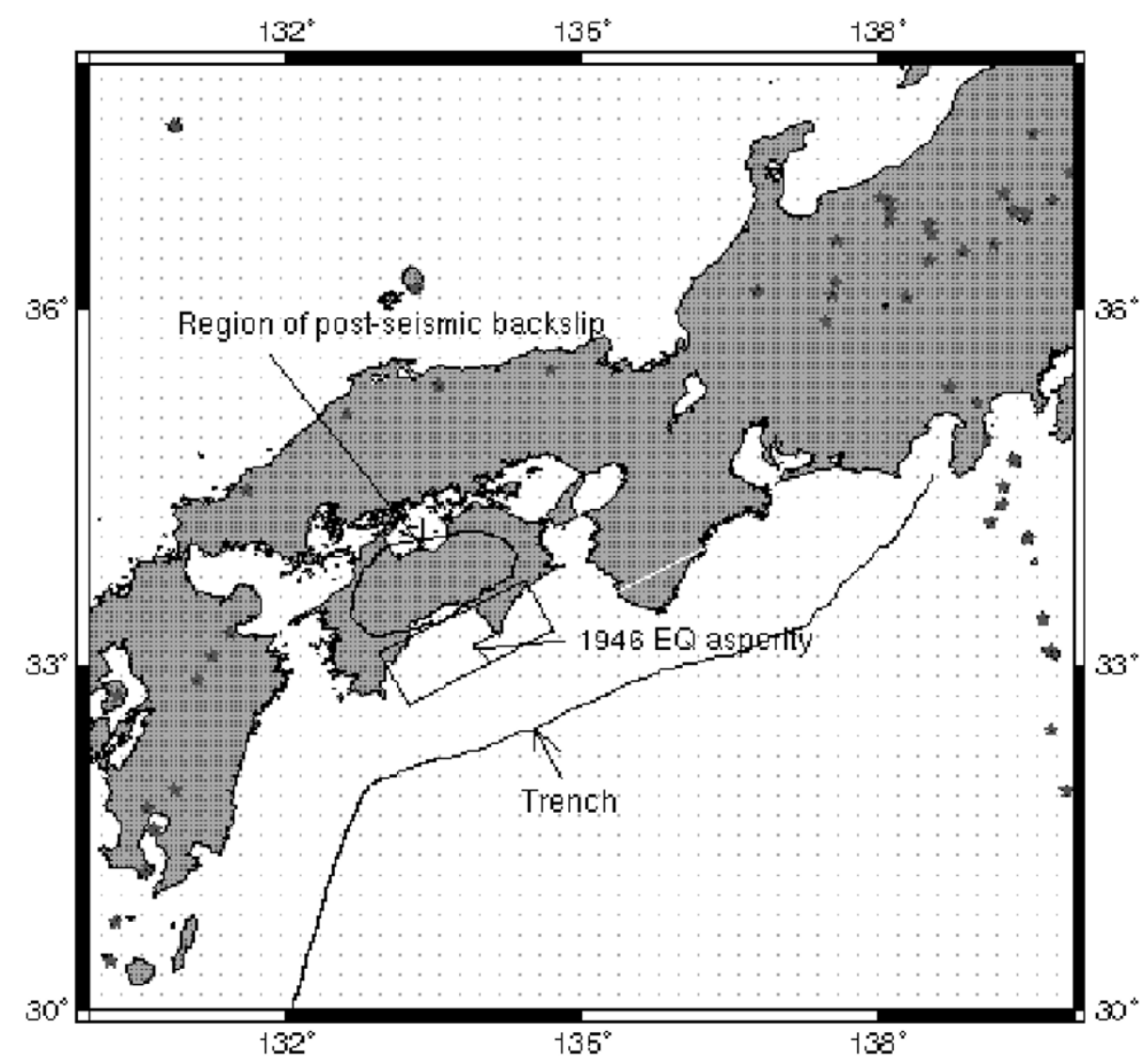

Fig. 1. Map of the Shikoku segment of the Nankai subduction zone, Japan. Several tectonic features are shown, including the main asperity of the 1946 great earthquake (Satake and Tanioka, 2001) and the portion of the deep creep plate interface that displayed rapid post-seismic creep after the 1946 earthquake (Sagiya, 1999).

law has a few parameters that must be specified to enable velocity weakening (stick-slip) within the seismogenic zone and velocity strengthening (creep) in the deeper zone. At the transition between regions, the interface behavior might be described as "conditionally stable" (see Scholz, 1990, for discussion).

Creep on the deep plate interface interacts with the shallow seismogenic zone via the elasticity of the surrounding rock. Steady creep increases the elastic shear stress on the locked portions of the seismogenic zone which eventually causes the large underthrusting earthquakes. Conversely, the static stress drop of these large earthquakes causes a step change in the stress state acting on the creeping interface. This interaction between the seismogenic and deep creep zones during the earthquake cycle has always been of great interest and there are many observational and theoretical studies. Most of the observational studies have focussed on just one subduction zone segment: the Shikoku segment of the Nankai zone (Fig. 1) where the Philippine Sea Plate subducts beneath Japan (Seno et al., 1993). In this paper, I will present yet another model of the seismogenic and creep zone interaction. But in contrast to most of the other papers on this topic, I shall focus on the simplest possible model of this interaction which produces analytic formulas that connect several key parameters to observations. There are a couple of surprising results that emerge from this simple model that should be tested by other more sophisticated models.

\subsection{Observations of slip in seismogenic and creep zones}

There is a great asymmetry in our observational constraints on the plate boundary. Seismologists have been able to determine several aspects of earthquake occurrence in the seismogenic portion. In a well-characterized zone such as the Nankai, the recurrence time of great earthquakes is known for several earthquake cycles (Ando, 1975), and the distribution of slip and static stress drop is well-determined for the last pair of great earthquakes that occurred in 1944 and 1946 (Ando, 1982; Satake and Tanioka, 2001). The methodology is well-developed for using slip across a finite fault in an elastic half-space to match co-seismic changes in stress and strain. On the other hand, all the seismological and geodetic studies cannot tell us the absolute level of shear stress acting on the seismogenic interface. We must use other more indirect methods, such as heat flow modeling (Tichelaar and Ruff, 1993; Peacock, 1996) or force balancing (Wang and He, 1999, and references therein), to infer something about absolute stress levels.

There are a few geodetic observations of post-earthquake strain changes that offer constraints on the character of the deep creep region. The advent of GPS methods and networks will certainly produce more and better observations in the future. At this time, the Nankai subduction zone is the best-studied example with some information on the preseismic, co-seismic, post-seismic, and inter-seismic phases of deformation (see Hyndman et al., 1995; and Sagiya and 
Thatcher, 1999, for review and discussion). At the risk of over-simplifying the picture, it is possible to summarize the results as follows: the pre-seismic accumulation is detected by surveys prior to the 1946 earthquake, but there is still some discussion over the spatial and temporal distribution of slip as to whether there was a creep event prior to the earthquake (Mogi, 1985); fault-averaged co-seismic slip of the 1946 Nankai event is about $4 \mathrm{~m}$ (Ando, 1982), though slip was concentrated at the down-dip edge in an asperity that is about $45 \mathrm{~km}$ wide and $180 \mathrm{~km}$ long (Satake and Tanioka, 2001; though Sagiya and Thatcher, 1999, offer a different solution); there was a rapid post-seismic slip in the deep creep plate interface just downdip of the asperity, the total accumulation of slip in this rapid phase is about $2 \mathrm{~m}$ (Savage and Thatcher, 1992) up to $3 \mathrm{~m}$ (Sagiya, 1999), and a quantitative fit to uplift data by Savage and Thatcher (1992) give a simple exponential function with a characteristic time of 4.7 years; the inter-seismic phase, from the 1950's to the present consists of steady linear motion in the deep creep segment while the 1946 main asperity remains locked to the subducting Philippine Sea plate and is moving toward $\mathrm{N} 50^{\circ} \mathrm{W}$ at a rate of about $45 \mathrm{~mm} /$ year with respect to Eurasia, consistent with the plate rotation pole of Seno et al. (1993), though there is considerable spatial variation across the seismogenic zone (see results in Sagiya, 1999; Ito et al., 1999; and Tabei, 1999).

\subsection{How to model seismogenic/deep creep interaction}

As argued by Savage (1995), all aspects of the geodetic results for the Nankai trough can be easily and successfully modeled by a fault interface in an elastic half space. Of course, we understand that the mantle beneath the lithosphere does display creep behavior and that this steady creep provides the basic plate tectonic velocity to the seismogenic region. This large-scale system of sinking slabs and mantle flow can be summarized by the kinematic boundary condition of plate interface slip velocity imposed at a depth close to the base of the lithosphere. Placing this kinematic boundary condition at a depth of $100 \mathrm{~km}$ or so is a good choice, given some geodetic results that measure plate motion across an entire subduction zone (e.g., Dixon, 1993; Sagiya, 1999; Tabei, 1999). If our main interest is in the strain changes associated with the earthquake cycle, then condensing all the complications of slab dynamics and mantle flow into an imposed velocity is clearly the best way to proceed. My simple model uses this kinematic condition to drive the system. In fact, my model is patterned after the Savage model, except that I replace the elasticity of the half-space with just two springs. At the end of the paper, I will argue that the Savage model can be modified with a different kinematic boundary condition that better describes the deep creep history in the earthquake cycle.

\section{The Model}

The fundamental model is a dipping plate interface embedded within an elastic half-space-the Savage model. Although many investigations are now using more complicated two- and three-dimensional models for the Nankai and other subduction zones (Thatcher and Rundle, 1984; Sato and Matsu'ura, 1992; Dmowska et al., 1996; Yoshioka, 1999; Hirahara, 1999; Stuart and Sagiya, 1999), my interest is in the basic interaction between the seismogenic and creep zones, hence a 2-D cross-section geometry is best. Since we know the subduction geometry and elastic moduli, the focus is on the constitutive laws that connect stress and slip across various portions of the interface. For the quasi-static treatment, earthquakes are represented by either the stress drop or co-seismic slip on the rupture area. Green's functions are available to calculate stress and strain everywhere in the 3-D elastic half space due to a change in slip or stress on any patch of the plate interface. In the 2-D model that I use, the plate interface is divided into two portions: the upper seismogenic zone where the constitutive law is stick-slip, and the deeper portion where the constitutive law is linear creep, i.e., where shear stress is proportional to slip velocity across the plate interface. With this plate interface division, one can still use the complete Green's functions, but I will group the elasticity response into just two springs, as illustrated in the lower part of Fig. 2. This simplification results in just two displacement functions, $u(t)$ and $v(t)$, and we can pretend that the associated strains are the observable space-averaged horizontal strains above the seismogenic and deep creep zones. One advantage of this discretization is that we can easily calculate the equivalent constant shear stress acting on each plate interface portion by dividing the interface force by the contact area, yet we still have a single displacement function between each block. When using the complete Green's functions, it is common to perform some spatial integration across each

(a) Seismogenic \& Deep Creep Plate Interface

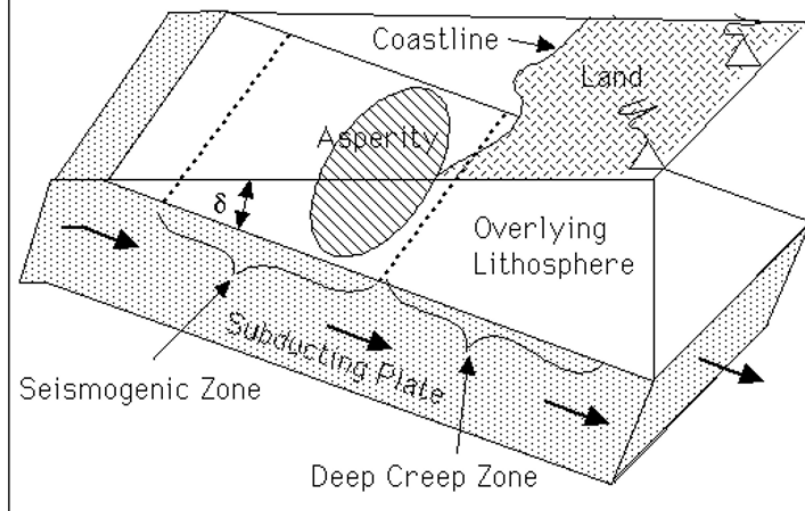

(b) Sketch of 2-D two-block mechanical model

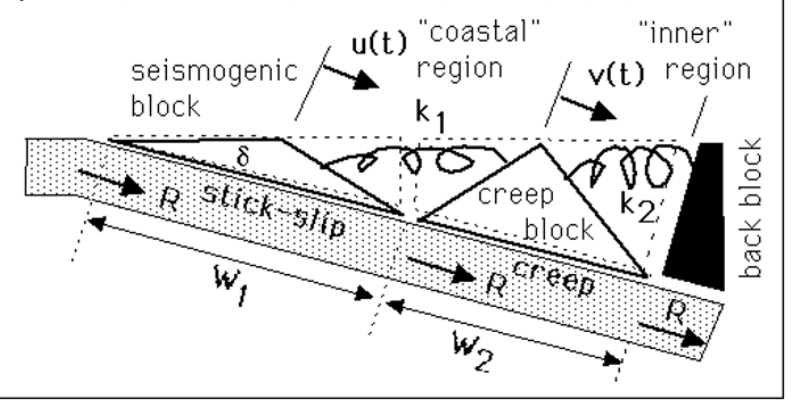

Fig. 2. View of geometry and plate interface in a subduction zone (top) with the 2-D two-block model that I use to examine seismogenic/deep creep interaction (below). 
fault patch to achieve a finite set of functions to evaluate (e.g., Stuart, 1988). Here, I just carry this procedure to its largest possible spatial scale. This same approach of using a small number of the largest possible blocks has already proven useful in studies of stress interaction within the seismogenic zone (e.g., Ruff, 1992; Nomanbhoy and Ruff, 1996). Although this two-block model for seismogenic/creep interaction is very simple, I give the complete development and all equations below so that others may easily use the final analytical formulas.

Model spring constants depend only on the geometry and elastic parameters of the overlying plate. Since we are using 2-D models, the force balance equations are written as force per unit length along the strike of the subduction zone. The spring constant units are then the same as for the shear modulus. The spring force equations are:

$$
\begin{aligned}
& f_{1}(t)=k_{1} u(t) \\
& f_{2}(t)=k_{2} v(t),
\end{aligned}
$$

where $f_{1}(t)$ and $f_{2}(t)$ are the spring force along the slab dip direction (per unit length along trench strike), and a positive force refers to outward directed force dipole. Thus a positive $f_{1}$ imparts a positive force to the creep block and a negative force to the seismogenic block. The displacement functions measure the contraction between the "seismogenic" and "creep" blocks (i.e. $u(t))$, and the "creep" and "back" blocks (i.e. $v(t)$ ). Referring to the geometry of Fig. 2, use the $W_{1}$ and $W_{2}$ length scales to discretize the elastic stress-strain relation for the block interaction in the overlying plate; then the spring constants per unit length can be written as:

$$
\begin{aligned}
& k_{1}=(8 / 3) \mu \sin \delta\left(2 W_{1} /\left(W_{1}+W_{2}\right)\right) \\
& k_{2}=(8 / 3) \mu \sin \delta\left(\left(W_{1}+W_{2}\right) / W_{2}\right)
\end{aligned}
$$

where $\mu$ is the overlying lithosphere shear modulus and a Poisson solid is assumed. Note that the ratio of spring constants for a constant dip plate interface and similar widths of the seismogenic and creep portions is two, i.e. $k_{2} / k_{1}=2$. Hence the $k_{2}$ spring is stiffer than $k_{1}$. This basic result is also easily obtained for a dipping fault in an elastic half-space, and it plays a key role for inter-seismic strain accumulation.

The linear creep law for the deep interface is:

$$
\sigma_{\text {creep }}(t)=\zeta s^{\prime}(t)
$$

where $s^{\prime}(t)$ is the slip velocity across the plate interface and $\zeta$ is the interface viscosity. For those more familiar with viscosity, $\eta$, we can view $\zeta$ as the effective viscosity across a uniformly shearing fault zone of width $j$, hence $\zeta=\eta / j$. However, since we cannot separate $\eta$ and $j$ from any observations, it is best to just use $\zeta$ as our fundamental physical property of the interface zone. Frictional constitutive laws also use this idea of an interface viscosity, though they typical use some non-linear connection between stress and slip velocity.

To translate the linear constitutive law of Eq. (3) into force per unit length along the subduction zone strike, we multiply both sides of (3) by $W_{2}$ to obtain:

$$
f_{\text {creep }}=W_{2} \zeta s^{\prime}(t)=W_{2} \zeta\left(R-v^{\prime}(t)\right)=\psi\left(R-v^{\prime}(t)\right)
$$

where we define $\psi$ to be $\left(W_{2} \zeta\right)$, and note that $s^{\prime}(t)$ equals $\left(R-v^{\prime}(t)\right)$ in the two-block model, where $v^{\prime}(t)$ is the derivative of $v(t)$.

The equation of motion is derived from the force balance on the two active blocks of the overlying plate. For the seismogenic block, the $k_{1}$ spring exerts a force in the negative direction for positive $u(t)$, and this force is transmitted to the plate boundary contact where it is balanced by an equal but opposite force across the interface. This force can increase until it reaches $f_{\text {fail }}$, then the "earthquake" happens which allows the seismogenic block to slip back and decrease the value of $u(t)$ by $\Delta u$ and the interface force by $\Delta f=k_{1} \Delta u$, which is related to earthquake stress drop. Three forces act on the creep block: the $k_{1}$ and $k_{2}$ spring forces, and the viscous force across the interface due to slip velocity mismatch across the interface, $s^{\prime}(t)=R-v^{\prime}(t)$. This force balance is:

$$
\begin{aligned}
& f_{1}+f_{2}+f_{\text {creep }}=0 \\
& k_{1} u(t)-k_{2} v(t)+\psi\left(R-v^{\prime}(t)\right)=0 .
\end{aligned}
$$

During any particular earthquake cycle, we have the kinematic constraint equation:

$$
(u(t)-u(0))+(v(t)-v(0))=R t
$$

where " $t$ " is now the time since the previous earthquake and $u(0)$ and $v(0)$ are the initial values at $t=0$. The velocity version of Eq. (6a) is:

$$
u^{\prime}(t)+v^{\prime}(t)=R
$$

where ' is used to denote the derivative with respect to time. Now use Eq. (6a) to eliminate $u(t)$ from Eq. (5), and the equation of motion reduces to just a single first-order ordinary differential equation for $v(t)$ :

$$
\begin{aligned}
v^{\prime}(t)= & R+k_{1}(u(0)+v(0)) / \psi+k_{1} R t / \psi \\
& -\left(\left(k_{1}+k_{2}\right) / \psi\right) v(t) .
\end{aligned}
$$

One of the key facts that we can extract from Eq. (7) is the characteristic creep time $\tau$ :

$$
\tau=\psi /\left(k_{1}+k_{2}\right)
$$

or equivalently:

$$
\tau=\zeta W_{2} /\left(k_{1}+k_{2}\right)
$$

With this substitution of $\tau$ back into Eq. (7), we can write the analytical solution for this simple differential equation as:

$$
v(t)=C+\left(k_{1} /\left(k_{1}+k_{2}\right)\right) R t+(v(0)-C) \exp (-t / \tau)
$$

where:

$$
C=R \tau\left(k_{2} /\left(k_{1}+k_{2}\right)\right)+\left(k_{1} /\left(k_{2}+k_{1}\right)\right)(u(0)+v(0)) .
$$

Recall that " $t$ " is the time within the earthquake cycle. We can use the kinematic constraint (Eq. (6)) together with Eq. (9) to obtain the solution for $u(t)$ and for the velocity functions $u^{\prime}(t)$ and $v^{\prime}(t)$.

The solution for $v(t)$ contains just three terms: a constant, a positive linear growth term, and an exponential decay term 
that can be positive or negative. A key aspect of the solution will be if the time to failure, $t_{\text {fail }}$, of the seismogenic block is less than or greater than $\tau$. If $t_{\text {fail }} \gg \tau$, then the exponential term decays to zero and we are left with a steady linear compression of the $k_{2}$ spring at a rate of $(R / 3)$ while the $k_{1}$ spring compresses at a rate of $(2 / 3) R$ (these specific rate numbers are for the case of $k_{2}=2 k_{1}$ ). But for times shorter than $\tau$, it is possible for the creep block to move trenchward if the $(v(0)-C)$ factor is negative and can overwhelm the linear compression term. Given the dependence of $(v(0)-C)$ on both initial values $u(0)$ and $v(0)$, we cannot say any more about the solution until we have some information about these initial values. Furthermore, we must regard $u_{\text {fail }}$ (or $t_{\text {fail }}$ ) as an unknown parameter.

\subsection{Constraints on $u_{\text {fail }}, u(0)$, and $v(0)$}

Our solution for $v(t)$ depends on three independent unknown parameters: $u(0), u_{\text {fail }}=u\left(t_{\text {fail }}\right)$, and $v(0)$. The initial and failure values for $u(t)$ depend directly on the final and failure stress of earthquakes. While seismologists cannot directly determine the absolute value of shear stress, it is possible to estimate the static stress drop, which can be translated into the force drop or displacement drop, $\Delta u=u_{\text {fail }}-u(0)$, of the simple mechanical model.

For the seismogenic interface, the earthquake stress drop average over the full seismogenic zone width is:

$$
\Delta \sigma=\Delta f_{1} / W_{1}=\left(k_{1} / W_{1}\right) \Delta u
$$

If we suppose that most of the co-seismic stress change occurs on the asperity part of the seismogenic interface, then we can write the asperity stress drop as:

$$
\Delta \sigma_{\text {asp }}=\Delta f_{1} / W_{\text {asp }}=\left(k_{1} / W_{\text {asp }}\right) \Delta u .
$$

We can use the basic seismological rule that static stress drops of large underthrusting earthquakes are a few MPa (Kanamori and Anderson, 1975) to fix the $\Delta u$ model parameter. Even better, observations of coseismic slip for the previous earthquake in a particular plate boundary segment specify the $\Delta u$ model value. We are still left with one unknown parameter value for either $u(0)$ or $u_{\text {fail }}$ that fixes the absolute stress level on the seismogenic interface. This value remains as a fundamental unknown in subduction zones, and hence also in this mechanical model.

We now turn to the $v(0)$ parameter. When you start this mechanical model, you can freely choose from a wide range of $v(0)$ values for the first earthquake cycle. After that, the $v(0)$ for the next earthquake cycle is the value of $v\left(t_{\text {fail }}\right)$ from the previous earthquake cycle. The mechanical system will evolve to find the long-time steady-state system cycle, whereby $v(0)$ will be the same from one cycle to the next. The system path in the $u(t)$ and $v(t)$ space then re-traces itself. The kinematic condition for this steady-state behavior is simply that $v(0)$ is the same from one earthquake cycle to the next, and can be written as: $v\left(t_{\text {fail }}\right)=v(0)$. I refer to this special $v(0)$ value as: $v_{s s}(0)$. Another way to write this kinematic condition is that the integral of $v^{\prime}(t)$ from $t=0$ to $t=t_{\text {fail }}$ must be zero. This condition implies that $v^{\prime}(t)$ is negative over part of the earthquake cycle. I have applied this kinematic constraint to Eq. (9) to find the analytical form for $v_{s s}(0)$. Then substitute this value for $v(0)$ back into the solution to obtain the final formula for $v(t)$ after any artificial start-up effects have decayed away:

$v_{s S}(t)=R \tau\left(1+\kappa_{1} \kappa_{2} J\right)+\kappa_{1} u(0)+\kappa_{2} R t+R \tau \kappa_{2} J \exp (-t / \tau)$

with:

$$
\begin{aligned}
\kappa_{1} & =\left(k_{1} / k_{2}\right) \\
\kappa_{2} & =k_{1} /\left(k_{1}+k_{2}\right) \\
J & =\left(t_{\text {fail }} / \tau\right) /\left(1-\exp \left(-t_{\text {fail }} / \tau\right)\right) .
\end{aligned}
$$

Since $\Delta u$ and $u_{\text {fail }}$ are the same for each earthquake cycle, the steady-state solution now gives a constant recurrence time between successive earthquakes, which is:

$$
T_{\text {recur }}=t_{\text {fail }}=\Delta u / R \text {. }
$$

Although the functional dependence of our steady-state solution is fairly simple, the algebraic expression in Eq. (11) makes it difficult to fully perceive system behavior. There is considerable algebraic reduction if we take a derivative to obtain the steady-state velocity function, $v_{s s}^{\prime}(t)$ :

$$
v_{s S}^{\prime}(t)=\kappa_{2} R[1-J \exp (-t / \tau)] .
$$

Note that we can easily construct $u_{s s}(t)$ and $u_{s s}^{\prime}(t)$ by use of the kinematic constraint equation (Eq. (6)). To probe the behavior of this steady-state solution a bit further, a key variable is the " $J$ " function. The numerical value of this function is plotted in Fig. 3 as a function of $\left(t_{\text {fail }} / \tau\right)$. This function is always greater than one. Thus at $t=0, v_{s s}^{\prime}$ will be negative: this is the post-seismic "backslip" in the deep creep region. We can imagine that $v_{s s}^{\prime}(t)$ would be the geodetically observed horizontal strain in the inner region, but most historical geodetic results are from the coastal region. Thus, there

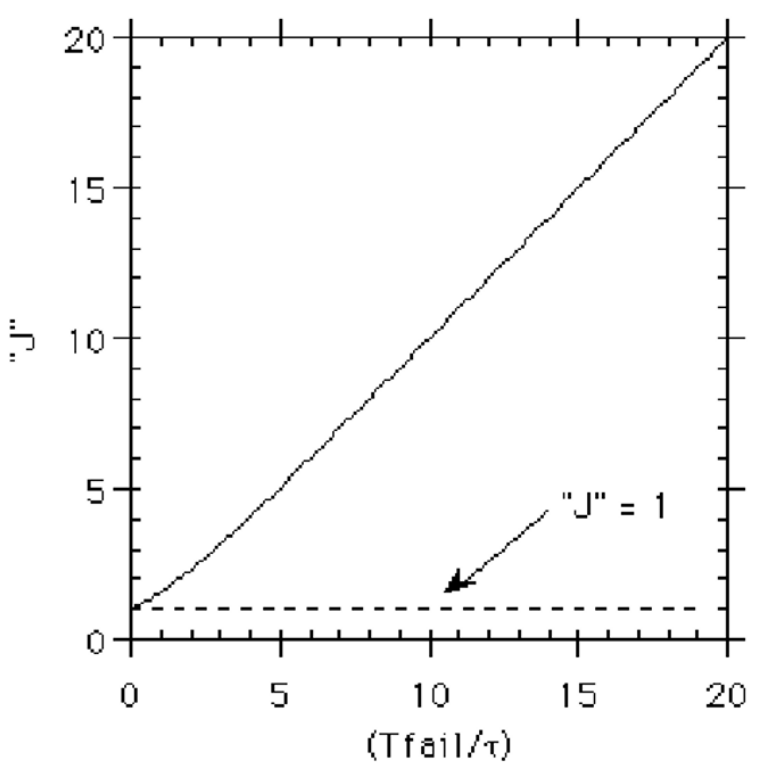

Fig. 3. Graph of the " $J$ " function that appears in the steady-state solution for $v_{s S}^{\prime}(t)$. " $J$ " is plotted as a function of the ratio between two times: great earthquake recurrence time and deep creep characteristic time $\left(T_{\text {recur }} / \tau\right)$. A value of 1 is shown by the dashed line. The fact that " $J$ " is 1 or larger implies that —in the steady-state solution — the creeper block moves trenchward immediately after a large earthquake. 


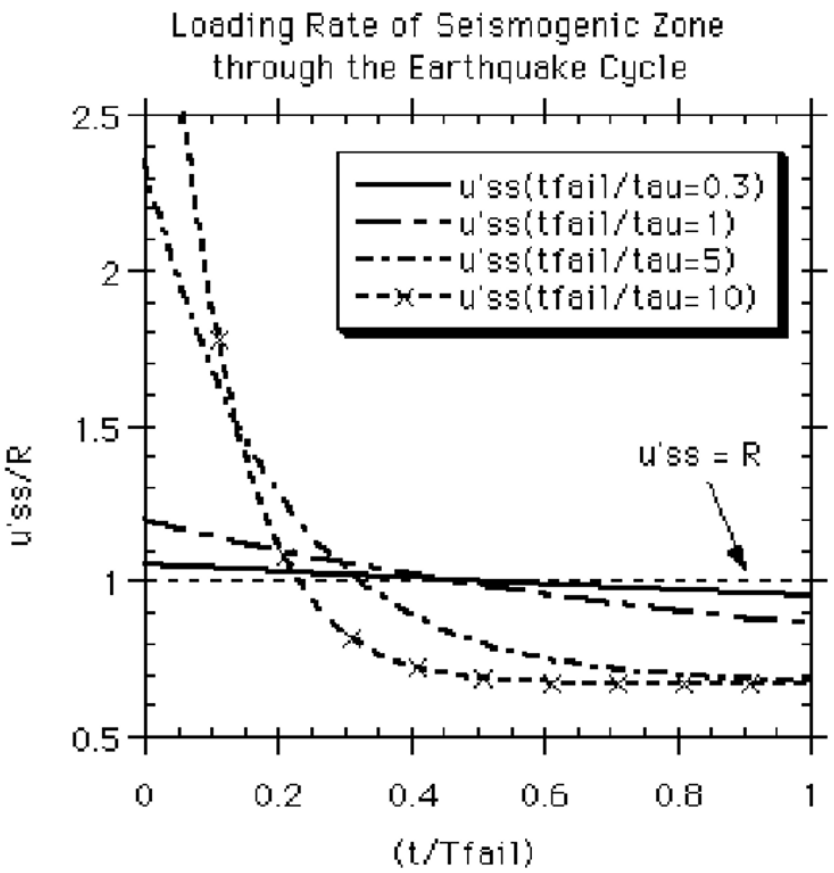

Fig. 4. Graph of $u_{s s}^{\prime}(t)$, which is related to horizontal strain rate in the "coastal" area, as a function of time through the earthquake cycle. Different $u_{s s}^{\prime}$ curves are for different values of the $\left(T_{\text {recur }} / \tau\right)$ ratio.

is greater interest in the $u_{s s}^{\prime}(t)$ function, plotted in Fig. 4 from $t=0$ to $t_{\text {fail }}$ for a variety of $\left(t_{\text {fail }} / \tau\right)$ values. We see that large values of $\left(t_{\text {fail }} / \tau\right)$ lead to a large and rapid post-seismic creep that re-loads the seismogenic zone to a significant fraction of its failure force. But note that this re-loading fraction is limited to about $(1 / 3)$ of the failure value even in the limit as $\left(t_{\text {fail }} / \tau\right)$ goes toward infinity. The remainder of the re-loading occurs as inter-seismic loading, which here can be defined as the contribution of the linear loading term in $u_{s s}^{\prime}(t)$. The analytical solution of Eq. (11) makes it easy to integrate the relative contributions of the post-seismic and inter-seismic terms. There is no need to graph the post-seismic reloading contribution as a function of $\left(t_{\text {fail }} / \tau\right)$, because it is identically equal to $\kappa_{2}$ for any and all values of $\left(t_{\text {fail }} / \tau\right)$ ! This is one of the more surprising theoretical results from this simple linear model. But if we translate this information to the practical world of strain observations, we will see that a distinction would be made between the extreme cases of $\left(t_{\text {fail }} / \tau\right) \ll 1$ and $\left(t_{\text {fail }} / \tau\right) \gg 1$.

Consider the extreme case of $\left(t_{\text {fail }} / \tau\right) \ll 1$, where the interface viscosity is relatively very high and the creep block acts more or less as a rigid extension of the back block. This situation concentrates the upper plate deformation to the coastal zone. Then $\left(v_{s s}^{\prime} / R\right)$ is always close to zero, plus and minus a little bit, while $\left(u_{s s}^{\prime} / R\right)$ is nearly 1.0 over the entire seismic cycle (see case of $t_{\text {fail }} /$ tau $=0.3$ in Fig. 4). In this case, the measured coastal horizontal strain would reflect a nearly linear increase at the full tectonic rate $R$, but the analytical formulas show that just $\left(1-\kappa_{2}\right) R$ of this rate is due to the linear inter-seismic term, the other $\kappa_{2} R$ nearlylinear contribution is the post-seismic term. Because the variation in $v_{s s}^{\prime}(t)$ is so small for the $\left(t_{\text {fail }} / \tau\right) \ll 1$ case, the strain observations might be consistent with a simple linear strain re-loading throughout the entire seismic cycle.

Now consider the other extreme where $\left(t_{\text {fail }} / \tau\right) \gg 1$, which implies that the interface viscosity is relatively low. The earthquake recurrence time is now much greater than the creep characteristic time, and the creep block experiences large motions during the earthquake cycle (see Fig. 4). There will be a rapid and large post-seismic adjustment with large "backslip" velocity, but just for a short time since $\tau$ is much smaller than $T_{\text {recur }}$. Then the motion of the creep block reverses and it compresses back during the inter-seismic interval. The proportion of the earthquake $\operatorname{sip}(\Delta u)$ that is reacquired in this post-seismic phase is exactly $\kappa_{2}$. As $\left(t_{\text {fail }} / \tau\right)$ becomes larger, the post-seismic backslip velocity becomes greater, but since the duration of this rapid post-seismic deformation is smaller, the integral effect is the same for all $\left(t_{\text {fail }} / \tau\right)$ values. Thus, unless you catch the post-seismic deformation just after the earthquake, all you would see is the linear re-loading of the seismogenic zone, albeit at an observed strain rate in the coastal region that is about $(2 / 3)$ - or in detail, $\left(1-\kappa_{2}\right)$ - the tectonic rate even though the seismogenic block is locked to the lower plate. The other part of the reloading was acquired very quickly in the post-seismic phase by the rapid motion of the creep block. A geodetic survey across the entire system would see the complete picture where the seismogenic block is in fact moving landward (with respect to the back block) at a constant rate of $R$, while the creep block is also moving landward at a rate of about $(1 / 3) R$. We shall return to this aspect later in the section where we consider a modified Savage model.

\section{Connection to Observations}

The simple two-block mechanical model cannot match any spatial details of observed deformation, but it might be able to match integrated quantities such as: ratios of earthquake recurrence time to characteristic creep time, ratio of integrated post-seismic back-slip to total slip, and segment averaged stresses. I will show that the observed value of $\left(t_{\text {fail }} / \tau\right)$ for the Shikoku segment allows us to infer other model parameters and make predictions for other observable ratios.

\subsection{Ratio of post-seismic slip to co-seismic slip}

The earthquake recurrence time for the Shikoku segment of the Nankai zone is variable: the last two intervals are 147 years and 92 years. By comparison, the characteristic time of rapid post-seismic creep is much shorter; e.g. Savage and Thatcher (1992) fit coastal uplift data with a combination linear reloading and rapid exponential reloading with a characteristic time of about 5 years. Thus, the observed ratio of $\left(t_{\text {fail }} / \tau\right)$ in the Shikoku segment is about 20 or so; clearly in the parameter space of $\left(t_{\text {fail }} / \tau\right) \gg 1$. If we choose model parameters to mimic the Shikoku segment geometry, $\kappa_{2}=(1 / 3)$, and the model predicts that the ( $\Delta u_{\text {post }} / \Delta u_{\text {coseismic }}$ ) ratio is about $(1 / 3)$. Average co-seismic slip is about $4 \mathrm{~m}$ (Ando, 1982), but $\Delta u_{\text {coseismic }}$ is about $6 \mathrm{~m}$ in the main asperity beneath the coast of Shikoku (Satake and Tanioka, 2001; though it may be as large as $11 \mathrm{~m}$, Sagiya and Thatcher, 1999). The total post-seismic "backslip" embodied in the geodetic analysis of Savage and Thatcher (1992) is less than $2 \mathrm{~m}$, and the ( $\left.\Delta u_{\text {post }} / \Delta u_{\text {coseismic }}\right)$ ratio from their uplift data is close to $(1 / 4)$. Another estimate of the integrated backslip is provided by Sagiya (1999) who estimated 
up to $3 \mathrm{~m}$ backslip. With the range in co-seismic and postseismic slip estimates, the $\left(\Delta u_{\text {post }} / \Delta u_{\text {coseismic }}\right)$ ratio ranges from about $(1 / 4)$ to more than $(1 / 2)$; and the predicted ratio of $(1 / 3)$ falls in the middle of this range. For any subduction zone segment similar to Shikoku with a relatively short characteristic time for deep creep, you should expect $\Delta u_{\text {post }}$ to be a substantial fraction of the co-seismic displacement.

Of course, there is more than just one model that can fit this pair of non-dimensionalized values. There is no doubt that more complicated models for the deep creep constitutive law could match these values (e.g., Stuart, 1988). Nonetheless, the main point here is that the simplest possible model of seismogenic and deep creep interaction can also approximately match the observed ratio of integrated post-seismic backslip in the deep creep zone to coseismic slip in the seismogenic zone.

\subsection{Interface viscosity in the deep creep segment}

Interface viscosity is an obscure property of the deep creep interface that depends on many hidden variables. One could extract some values from the rate-and-state friction constitutive law if you are willing to extrapolate the internal parameters down to the deep creep conditions. Indeed, simulations of seismogenic/creep zone interaction such as those by Stuart (1988) and others must calculate the effective $\zeta$ inside their computer programs. What we really need to do is find some way to measure $\zeta$ with observations-we can do that! Glancing back at the solution for $v(t)$ (Eq. (11)), we see that $\psi$ only affects the deformation through its appearance in $\tau$. Recall that:

$$
\tau=\psi /\left(k_{1}+k_{2}\right),
$$

and we re-arrange this to solve for $\zeta$ :

$$
\zeta=\tau\left(k_{1}+k_{2}\right) / W_{2}
$$

Given that $W_{2}, k_{1}$, and $k_{2}$, are set by subduction zone geometry, the main challenge is to observe $\tau$. The best way to obtain a good estimate for $\tau$ is to measure the characteristic time of the deep creep post-seismic transient. As discussed above, that has been done for the Shikoku segment with the result that $\tau$ is about 5 years.

Thus, with $\tau$ now directly measured, the only other variable in Eq. (14) is $\zeta$. We can substitute for $k_{1}$ and $k_{2}$ to have an explicit formula for $\zeta$ that depends on the subduction zone geometry and elasticity:

$$
\begin{aligned}
\zeta= & \tau((8 / 3) \mu \sin \delta)\left(2 W_{1} W_{2}+\left(W_{1}+W_{2}\right)^{2}\right) / \\
& \left.\left(W_{2}^{2}\left(W_{1}+W_{2}\right)\right)\right)
\end{aligned}
$$

and for the special case when $W_{1}=W_{2}$, the formula is:

$$
\zeta=\tau(8 \mu \sin \delta) / W_{2} \quad\left(W_{1}=W_{2}\right) .
$$

The units of $\zeta$ are $\langle$ stress/velocity $\rangle$, and hence the SI units will be $\langle\mathrm{Pa} /(\mathrm{m} / \mathrm{s})\rangle$. The units of this interface viscosity also can be viewed as $\langle\mathrm{Pa} \mathrm{s} / \mathrm{m}\rangle$, and interpreted as $\langle$ bulk viscosity/interface thickness $\rangle$. But it is more practical to think of $\zeta$ as an interface property that gives the slip velocity response to an imposed shear stress across the interface.

Now, lets calculate the numerical value of $\zeta$ for the creep portion of the plate interface beneath Shikoku. With $W_{2}=$
$150 \mathrm{~km}$ and $\mu=5 \times 10^{10} \mathrm{~Pa}$ (compromise between crust and mantle values), and $\tau=5$ years, we find:

$$
\zeta=8.4 \times 10^{13} \mathrm{~Pa} /(\mathrm{m} / \mathrm{s}) .
$$

To my knowledge, this is the first time that the interface creep viscosity has been estimated directly from observations in Shikoku, or in any other subduction zone. Note that other investigators of seismogenic/creep zone interaction must have used some comparable value in their calculations, but they have chosen not to emphasize this aspect of their studies. In the context of the simple linear model, it is remarkably easy to "invert" the observed value for $\tau$ into a value for interface viscosity. The theory is certainly trivial and it is easy to specify the subduction zone geometry, but finding a good estimate for $\tau$ is the difficult part. Here, I have used the results of 50 years of geodetic measurements in the Nankai region as analyzed by a long list of distinguished investigators. In a later section, I shall calculate $\zeta$ for the Alaska and Chile subduction zones based on less-scrutinized estimates for $\tau$. A challenge for the future is to obtain good estimates for $\tau$ in many other subduction zones.

\subsection{Stress in the deep creep plate interface}

The absolute stress level in the seismogenic zone over the earthquake cycle must fall between $\sigma_{o}$ and $\sigma_{\text {fail }}$; which for the two-block model is set by the choice of either $u(0)$ or $u_{\text {fail }}$. Thus, the seismogenic interface average stress level is an "external" parameter. In contrast, the stress level at the creep zone interface is determined internally by the system as its finds the stable system path. The creep interface force is proportional to slip velocity across the interface. Thus, the $k_{1}$ and $k_{2}$ springs could both exert a high force on the creep block, but if these elastic forces balance, then this high level of background stress has no effect on the creep interface stress level. So what does determine the stress level on the creep interface?

During the earthquake cycle, there can be a large variation of force on the creep interface, though the force will always be directed landward for the steady-state solution. If the interface is weak (small $\zeta$ ) so that the subducting plate can slide past with only small stresses at the interface, then the shear stress would be small during most of the earthquake cycle. But just after the earthquake occurs, force balance on the creep block makes it "backslip" at a fast enough rate to generate a creep interface stress that is comparable to the earthquake stress drop. Thus, for the case of a very weak creep interface, the average stress over the earthquake cycle will be somewhere between zero and the earthquake stress drop. At the other extreme, if the creep interface viscosity is very high such that high stresses are required to make the overlying plate slip across the subducting plate, then the system will evolve to where the $k_{2}$ spring is much more compressed than the $k_{1}$ spring. This elastic force mismatch can then balance the viscous drag force. In the extreme limit where the seismogenic block forces are tiny compared to this viscous drag, then the steady state solution will be that $v^{\prime}(t)$ is zero throughout the earthquake cycle and the slip velocity at the deep creep interface will be about $R$. Hence the creep interface force will be about $\psi R$. For the above extreme cases, the average creep zone force will either fall between 0 and $\Delta f$, or be about $\psi R$. 
Lets now consider the general solution to the two-block model and look further into the average creep interface stress. Given the steady-state solution for $v_{s s}^{\prime}(t)$ in Eq. (13), it is straightforward to construct the force acting across the deep creep interface through the earthquake cycle:

$$
f_{\text {creep }}(t)=\psi\left(R-v_{s s}^{\prime}(t)\right)
$$

then integrate this force from $t=0$ to $t=t_{\text {fail }}$ and divide by $t_{\text {fail }}$ to find the average creep force. Since we can do this analytically, we can then view the explicit dependence of the average force on all the system variables. But the answer is:

$$
f_{\text {creepAV }}=\psi R
$$

Yes, the deep creep interface force averaged over the earthquake cycle is completely independent of all other parameters. The easiest way to see that this result must be true is to consider the kinematic constraint condition on $v_{s s}^{\prime}$. Given the above discussion, there is no surprise that $f_{\text {creepAV }}$ is $(\psi R)$ for parameter choices that make $\left(t_{\text {fail }} / \tau\right) \ll 1$, but the curious result is for the case of a weak creep zone such that $f_{\text {creepAV }}$ should be between zero and the earthquake force drop. It turns out that $(\psi R)$ must be precisely within this range at the correct quantitative value to equal $f_{\text {creepAV}}$. Of course, this cannot be a coincidence. We will first explore this facet, and then make use of it in the next section.

While we can analyze the system equations to derive $f_{\text {creepAV }}=\psi R$, perhaps the best insight is gained by examining the graphical solution for $u(t)$ and $v(t)$ at steadystate (Fig. 5). Two key iso-force lines are drawn in this graph. When the spring forces are equal and opposite (for $\left.v=\left(k_{1} / k_{2}\right) u\right)$, then the creep block force balance requires that $v^{\prime}=R$ and $u^{\prime}=0$. Hence, the system cannot follow a path along this iso-force line. The arrows in Fig. 5 show the direction of system trajectories from this iso-force line point "up". The other key iso-force line is for a net spring force on the creep block of $(-\psi R)$ in the trenchward direction. This requires a viscous drag force at the creep interface of $(+\psi R)$, which means that $v^{\prime}=0$. Given that the seismogenic block moves landward at a rate of $R$, the $k_{1}$ spring will continue to compress which will move the system off this iso-force line. The system trajectories are plotted in Fig. 5 and they point to the "right". From these considerations, it is clear that the mechanical system will be attracted to some path between these two iso-force lines. The earthquake will occur when the system path hits the vertical line for $u_{\text {fail }}$, then $v$ remains the same while $u$ jumps back by $\Delta u$. A complete steady-state earthquake cycle path is shown. Note that the system is attracted to a path such that the earthquake jump places it above the $v^{\prime}=0$ iso-force line: $v^{\prime}$ is negative above this iso-force line, and this trajectory is the post-seismic phase of backslip. Once the system crosses the $v^{\prime}=0$ iso-force, the trajectory points toward $+v$ and $+u$ and it follows a nearly linear trend until it hits $u_{\text {fail }}$ again. Figure 5 shows two choices for $u_{\text {fail }}$, where all other system variables are the same. While the average force level on the seismogenic interface depends directly on this "external" choice for $u_{\text {fail }}$, the steady state system path is balanced about the $v^{\prime}=0$ iso-force line for any choice of $u_{\text {fail }}$. Figure 5 clearly shows the force acting on the creep interface varies about the value of $(\psi R)$ - and then the analytic solution shows that it is exactly $\psi R$.

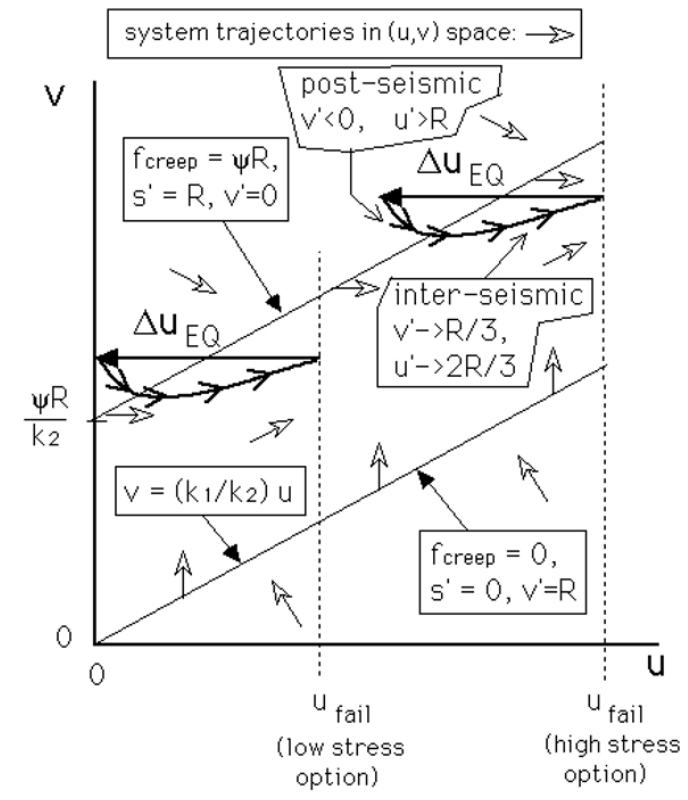

Fig. 5. Plot of creep block displacement $(v)$ versus seismogenic block displacement $(u)$. Two iso-force lines are shown, with the resultant instantaneous block velocities indicated. Open-headed arrows show instantaneous system trajectories at various points in $(u, v)$ space. The "earthquake" occurs when $u$ increases to the failure value, $u_{\text {fail }} . \Delta u$ is the displacement jump associated with earthquake stress drop. The bold lines and curves schematically plot the steady-state earthquake cycle paths for two different choices of $u_{\text {fail }}$. Note that for a steady-state system, creep interface force varies about the $(\psi R)$ iso-force line for any choice of $u_{\text {fail }}$.

System path trajectories in Fig. 5 show that the system will be attracted to its steady-state path no matter what initial values we might choose for $u$ and $v$; though it may take many earthquake cycles. Other investigators who use sophisticated models of seismogenic/creep interaction must allow those models to evolve over many earthquake cycles to find their long-term solution, otherwise you have a model that is still dominated by its transient response to the un-natural humanimposed initial conditions. The time lost for system evolution can be reduced by "pre-stressing" the model to make the deep creep zone stress compatible with the constitutive law and plate tectonic rate.

\subsection{Average creep interface stresses from observations}

We now turn to another facet of the result that average creep force is exactly $(\psi R)$. As discussed above, we cannot find the absolute stress on the seismogenic plate interface based on seismological/geodetic observations, but the surprising result is that we CAN measure the absolute stress for the deep creep plate interface! The first step in this procedure is described in the above section where we realize that creep interface force is independent of the background stress level acting through the lithosphere above it. In other words, deep creep interface stress is independent of the absolute stress level acting on the seismogenic plate interface. Furthermore, the average stress over the earthquake cycle has the surprisingly simple formula derived above:

$$
f_{\text {creepAV }}=\psi R \text {. }
$$

We can rewrite this formula (see Eq. (3)) for the average shear stress acting on the deep creep interface over the earthquake 
cycle as:

$$
\sigma_{\text {creepAV }}=\zeta R
$$

Given that we know $R$, it is trivial to calculate $\sigma_{\text {creepAV }}$ if we know $\zeta$-and we do know it. In Subsection 3.3 above, I derived a simple formula that uses the $\tau$ estimate in the Shikoku segment to calculate $\zeta$. All we need to add is the plate tectonic velocity of $45 \mathrm{~mm} /$ year (Seno et al., 1993) to calculate a "direct" estimate of the average absolute level of shear stress acting on the deep creep portion of the Shikoku subduction zone:

$$
\left.\sigma_{\text {creepAV }}=1.2 \times 10^{5} \mathrm{~Pa} \quad \text { (or, } 1.2 \text { bars }\right)
$$

While many other studies of seismogenic/creep zone interaction in the Nankai must have a similar stress estimate internal to their numerical calculations, this is (to my knowledge) the first "direct" estimate of creep interface stress based on observations and a simple model. The plate interface stress level of a few bars is a familiar number to many geophysicists because it is just about the same value as earthquake stress drops for large underthrusting earthquakes.

Let me review the procedure to obtain absolute shear stress estimates in the deep creep plate interface in subduction zones: (i) find a geodetically measured value of $\tau$, the characteristic time for post-seismic backslip in the deep creep interface; (ii) look at a cross-section of the subduction zone geometry to obtain $\delta, W_{1}$, and $W_{2}$; (iii) then calculate $\zeta$, the creep interface viscosity, with Eq. (14); (iv) and then use this value of $\zeta$, the plate tectonic rate, $R$, and in Eq. (16), to find $\sigma_{\text {creepAV. }}$. The simple model that I have used produces algebraic relations that are easily manipulated to calculate the absolute stress level on the plate boundary. In a section below, we will consider the robustness of this simple procedure.

\subsection{Ratio of deep creep stress and earthquake stress drops}

Our creep interface stress estimate in the above section is at the lower end of observed earthquake static stress drops (Kanamori and Anderson, 1975), yet within the range of observed quasi-dynamic stress drops for underthrusting earthquakes (Ruff, 1999). If we reflect back on the qualitative discussion in Subsection 3.3 and recall that $\left(t_{\text {fail }} / \tau\right) \gg 1$, we would expect that the creep interface stress in Nankai should fall between zero and the earthquake stress drop. In fact, we can manipulate Eqs. (16), (8), and (10) to derive an expression for the ratio of $\sigma_{\text {creepAV }}$ to model earthquake stress drop, $\Delta \sigma_{\mathrm{EQ}}$ :

$$
\left(\sigma_{\text {creepAV }} / \Delta \sigma_{\mathrm{EQ}}\right)=\left(W_{1} / W_{2}\right)\left(1 / \kappa_{2}\right)\left(\tau / t_{\text {fail }}\right) .
$$

Given our assumed geometry and the measured $\left(\tau / t_{\text {fail }}\right)$ ratio of $(1 / 20)$ for the Shikoku region, Eq. (18) predicts that the $\left(\sigma_{\text {creepAV }} / \Delta \sigma_{\mathrm{EQ}}\right)$ ratio is about $(1 / 7)$. Then given the numerical value of $\sigma_{\text {creepAV}}$, the model prediction for $\Delta \sigma_{\mathrm{EQ}}$ is about $8.4 \times 10^{5} \mathrm{~Pa}$ (or, 8.4 bars).

We can calculate the model stress drop of the 1946 earthquake in the Shikoku zone from the results of Ando (1982) or Satake and Tanioka (2001). Ando (1982) gives an average displacement of about $4 \mathrm{~m}$ over the entire fault area. If we use Eq. (10) with $W_{1}=150 \mathrm{~km}, \Delta u_{\mathrm{EQ}}=4 \mathrm{~m}$, and $\mu=5 \times 10^{10}$
$\mathrm{Pa}$, then the model stress drop is:

$$
\Delta \sigma_{\mathrm{EQ}}=7.1 \times 10^{5} \mathrm{~Pa} .
$$

On the other hand, if we estimate the asperity stress drop from the parameters in Satake and Tanioka (2001), we find that:

$$
\Delta \sigma_{\text {asp }}=35 . \times 10^{5} \mathrm{~Pa} .
$$

As expected, the model stress drops agree reasonably well with the predicted value from Eq. (18). Note that these model stress drops refer to the simplistic 2-D representation of the overlying plate elasticity; others might derive slightly different numbers for the more correct continuum 3-D model.

Could it be a coincidence that the creep stress is within an order of magnitude of earthquake stress drop? The creep stress directly depends on creep interface viscosity and plate tectonic velocity. Thus, if we seek some deeper understanding of the near-coincidence of the stress drop and creep stress, then it must come from an examination of what controls earthquake stress drop and creep interface viscosity. Could the order-of-magnitude equivalence of stress drop and creep stress be a consequence of a unified constitutive law? The answer to this question is beyond the scope of this paper.

\subsection{Application to Alaska and Chile}

Given how easy it is to estimate a model interface viscosity and creep interface stress, it is tempting to apply this procedure to other subduction zones. However, we must remind ourselves that it is difficult to extract a reliable estimate of the post-seismic creep characteristic time. Despite this warning, I shall proceed to estimate $\zeta$ and $\sigma_{\text {creepAV }}$ at two other subduction zones. Previous studies have found evidence for post-seismic deep creep after the two greatest earthquakes of the 20th century: the 1960 Chilean $(M w=9.5)$ and 1964 Alaskan $(M w=9.2)$ earthquakes.

3.6.1 Alaska There is good evidence of co-seismic uplift and subsidence in and around the 1964 Alaska earthquake (Plafker, 1972), mostly due to the clever use of natural sea level markers. In addition, many follow-up studies and geodetic work have provided some evidence of rapid postseismic creep and its characteristic time. As summarized in Brown et al. (1977) and Savage et al. (1998), the best estimate we can make is that the post-seismic rapid creep had a characteristic time of about 5 years-quite similar to the Shikoku value - and that the total slip in the deep creep zone over this time was about $2.3 \mathrm{~m}$. While there are many models of co-seismic slip for the 1964 earthquake, I will use the overall average value of $12 \mathrm{~m}$ from Brown et al. (1977) for the sake of internal consistency. Of course, the only geodetic observation we need to calculate both $\zeta$ and $\sigma_{\text {creepAV }}$ is $\tau$, but I will also check the $\left(\Delta u_{\text {post }} / \Delta u_{\text {coseismic }}\right)$ ratio for compatibility with the model. Since the subduction zone geometry and size varies from Nankai to Alaska, I use the Alaskan geometry shown in Fig. 6 with $\mu=5 \times 10^{10} \mathrm{~Pa}$ (the geometry is from Brown et al., 1977). Now, put the Alaska values for $W_{1}, W_{2}, \delta$, and $\tau$ into Eq. (14b) to obtain:

$$
\zeta_{\text {Alaska }}=1.6 \times 10^{14} \mathrm{~Pa} /(\mathrm{m} / \mathrm{s}) .
$$

This creep viscosity estimate is about twice the value we found for Nankai even though $\tau$ is the same; the difference is 


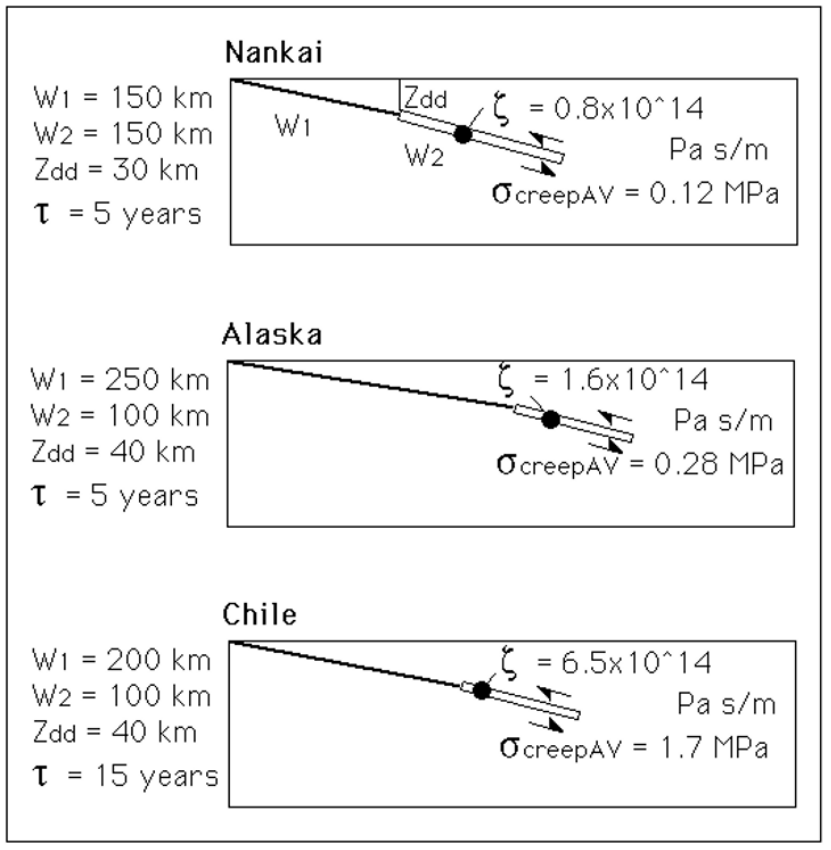

Fig. 6. Model predictions for interface viscosity and deep creep zone stress for Nankai, Alaska, and Chile subduction zones. Cross-section sketches show the subduction zone geometry used to calculate the $k_{1}$ and $k_{2}$ constants. Absolute shear stress estimates for the creep interface are slightly less than earthquake stress drops.

mostly due to the smaller $W_{2} / W_{1}$ ratio for Alaska. We now use a plate tectonic rate of $55 \mathrm{~mm} /$ year at Alaska to give a deep creep stress estimate of:

$$
\sigma_{\text {creepAV } \rightarrow \text { Alaska }}=2.8 \times 10^{5} \mathrm{~Pa} \quad(2.8 \text { bars }) .
$$

Since the rate is about the same for Nankai and Alaska, the factor of two increase in stress level for Alaska is mostly due to the larger $\zeta$ estimate.

Newer models of Alaska co-seismic and inter-seismic deformation (e.g., Savage et al., 1998) use a subduction zone geometry that differs from what I used in Fig. 6. However, the key variable is $\tau$. If the $\tau$ estimate remains at around 5 years, then the above numbers will not change too much.

There is still some controversy over the recurrence time for the great Alaska earthquake, but it is longer than 100 years. Thus, the model system ratio $\left(t_{\text {fail }} / \tau\right)$ is much larger than 1 , and we would expect to see that the geodetically measured ratio of $\left(\Delta u_{\text {post }} / \Delta u_{\text {coseismic }}\right)$ is $(1 / 3.4)$. The measured value of this ratio (from Brown et al., 1977) is (2.3/12), or about $(1 / 5)$. This estimate falls just out of the range of various estimates from Nankai.

3.6.2 Chile Given the coseismic fault displacements of $20 \mathrm{~m}$ or more, there is considerable natural evidence for the pattern of coseismic uplift and subsidence for the great 1960 Chile earthquake (Plafker and Savage, 1970). Geodetic surveys are somewhat sparse in Chile, so there is poor spatial control on subsequent post-seismic deformation. Barrientos et al. (1992) used two tide gauge stations-one at the edge and one in the middle of the rupture zone- to detect and quantify post-seismic slip in the deep creep interface. The post-seismic deformation could be reasonably fit by a single exponential function with a characteristic time of 15.6 years, though Barrientos et al. (1992) also show that a quadratic curve could fit the data. They modeled the post-seismic creep by extending the edge of the creeping zone to deeper depths through time. I can use their summary model parameters where they found the total slip in the postseismic deformation phase is about 3 to $5 \mathrm{~m}$ over an interface width of about $100 \mathrm{~km}$.

I use the Chile geometry shown in Fig. 6 with $\mu=5 \times 10^{10}$ $\mathrm{Pa}$ (the geometry is from Barrientos et al., 1992). Now, put the Chile values for $W_{1}, W_{2}, \delta$, and $\tau$ into Eq. (14b) to obtain:

$$
\zeta_{\text {Chile }}=6.5 \times 10^{14} \mathrm{~Pa} /(\mathrm{m} / \mathrm{s}) .
$$

This creep viscosity estimate is about a factor of eight larger than the value we found for Nankai; the increase is due to a combination of the larger $\tau$ and the assumed geometry. We now use a plate tectonic rate of $84 \mathrm{~mm} /$ year at Chile to find a deep creep stress estimate of:

$$
\sigma_{\text {creepAV } \rightarrow \text { Chile }}=17.3 \times 10^{5} \mathrm{~Pa} \quad(17.3 \text { bars }) .
$$

The factor of ten increase in stress level for Chile compared to Nankai is mostly due to the larger $\zeta$ estimate.

For Chile, the great earthquake recurrence time is more than about 120 years. Thus we have a $\left(t_{\text {fail }} / \tau\right)$ ratio of more than 8 , which again implies that observed post-seismic slip in the creep zone should be about (1/3.3) of the coseismic amount. Estimates of co-seismic slip for the Chile earthquake vary from about $20 \mathrm{~m}$ up to possibly $40 \mathrm{~m}$ (see Barrientos et al., 1992, for discussion and references), hence the observed ratio of $\left(\Delta u_{\text {post }} / \Delta u_{\text {coseismic }}\right)$ is bounded by $(1 / 13)$ to $(1 / 4)$. Clearly, this number should be more refined before we can claim that the simple model matches-or not- the $\left(\Delta u_{\text {post }} / \Delta u_{\text {coseismic }}\right)$ ratio in Chile.

\section{Further Testing of the Model Stress Estimates}

The simple two-block model provides formulas to estimate the plate interface viscosity and the absolute stress state on the deep creep part of the plate boundary. The results seem quite reasonable, so it is tempting to accept these numbers for stress. But before we do so, we must consider the robustness of these results. How sensitive are the final numbers to small changes in other model parameters or model behavior? The sensitivity to model parameters is directly seen in the simple formulas. Since we do know subduction zone geometry and basic elastic constants quite well, there is no significant source of error there. On the other hand, the simplicity of the model enforces a uniform creep over the entire downdip length of $W_{2}$. Realistic deep creep models (e.g., Stuart, 1988) show that the post-seismic creep decays with depth. Hence, the post-seismic characteristic time measures the creep viscosity of the upper part of the deep creep interface. To probe the impact of this effect, we can perturb the simple model by concentrating all the plate boundary stress into a smaller width $\left(W_{\text {small }}\right)$, similar to using asperities in the seismogenic zone to concentrate the stress drop. Since we must convey the same force across the plate boundary, both the interface viscosity and interface stress will increase by the factor of $W_{2} / W_{\text {small }}$. How small can $W_{\text {small }}$ be? Again, probably the best constraints are from the Shikoku region. The spatial-temporal modeling of Sagiya (1999) shows that 


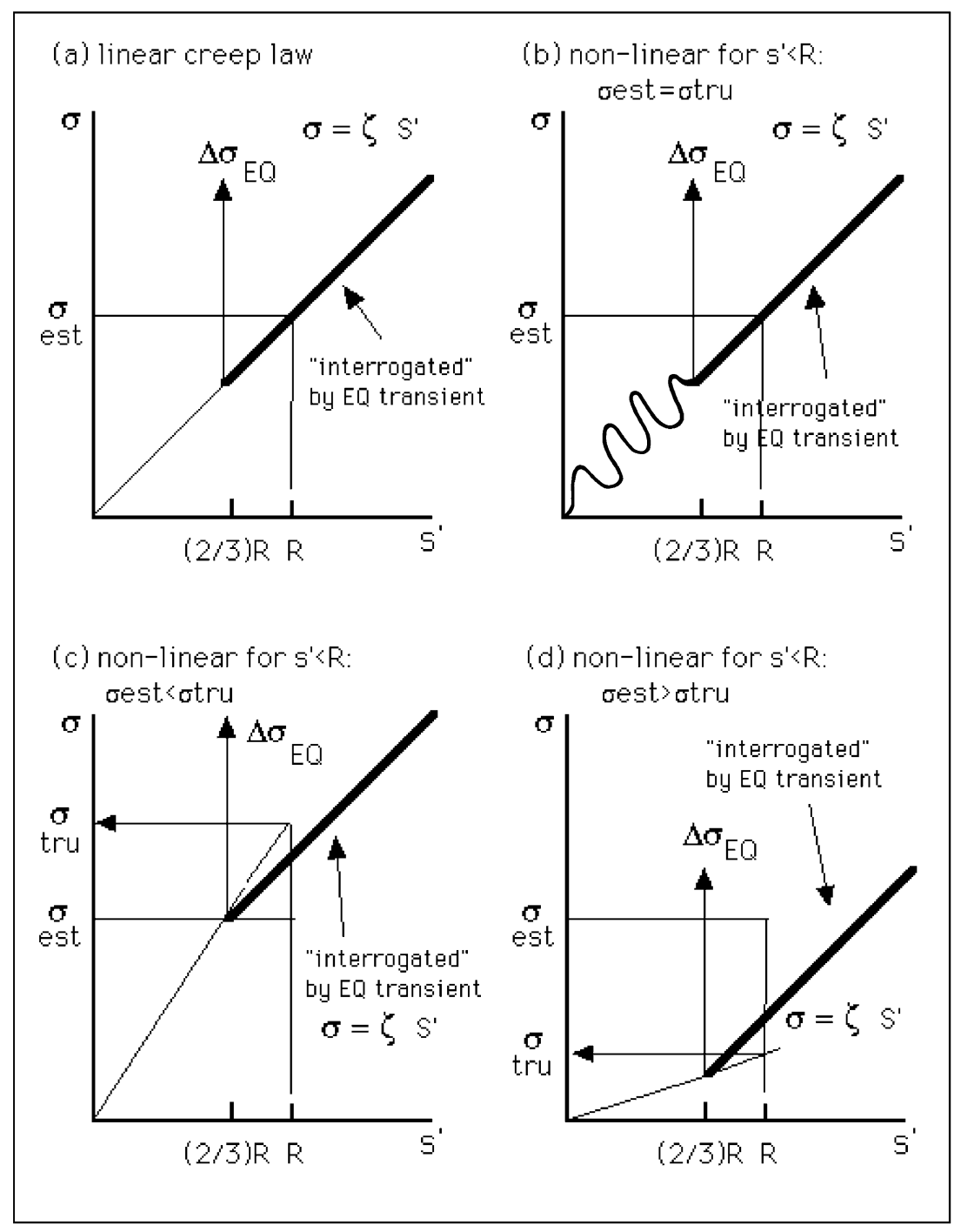

Fig. 7. Robustness of deep creep stress estimate. The assumed linear creep law is shown in (a), the earthquake stress drop interrogates the constitutive law over the stress/creep velocity range shown as the bold line. (b) through (d) show non-linear variations in the creep law for velocities less than (2/3)R, and compares the "true" absolute shear stress $\left(\sigma_{\text {tru }}\right)$ to the stress estimate $\left(\sigma_{\text {est }}\right)$ from (a).

the post-seismic creep is consistent with a downdip width of the creep zone of at least $50 \mathrm{~km}$. Most continuum theoretical models produce post-seismic slip that decays in the downdip direction with a characteristic distance related to the seismogenic zone width; hence a creep zone width of 50 to $150 \mathrm{~km}$ is grossly compatible with the predictions of any smoothly varying continuum model. If the resistive creep stress in Shikoku is concentrated in the upper third of the assumed deep creep zone, then $\sigma_{\text {creepAV }}$ increases from 1.2 to 3.6 bars. If we also change the length scale for the elasticity constants, then $\sigma_{\text {creepAV }}$ can increase to 6.6 bars. In conclusion, we anticipate that models more accurate than the two-block model might produce larger values for both $\zeta$ and creep interface stress, but they should still agree to within a factor of five or so.

Another potential source of error in these estimates would be a non-linear constitutive law for the creep zone. The creep law might follow a simple power law between stress and slip velocity, or it may require much more complicated friction laws such as those discussed earlier. Given the evidence for a "creep event" somewhere in either the seismogenic or deep creep zone before both the 1946 earthquake (Mogi, 1985) and the 1960 Chile earthquake (Kanamori and Cipar, 1974; Cifuentes and Silver, 1989), we must ponder the consequences of non-linear creep laws. While some choices for non-linear creep will not significantly affect the major conclusions here, other choices could change our model estimates of absolute stress in the creep region. It is difficult to find useful analytical solutions for most non-linear creep laws, but it is easy to see how various classes of non-linear creep laws would affect our results. We obtain the creep viscosity and the absolute stress level of the creep interface in a two-step process. Figure 7(a) shows the situation for a linear creep law. Basically, the stress perturbation from the earthquake "interrogates" the shape and slope of the interface constitutive law over the range shown. A linear creep law results in the simple exponential decay back to the pre-seismic stress level. Observing the characteristic time of this decay response then fixes the slope of the stress versus velocity relation (i.e. interface creep viscosity). Then, we use this slope to find the value of abso- 
lute stress associated with the plate tectonic rate, $R$. Given that all post-seismic creep observations are roughly consistent with a linear creep law over the "interrogated" range, I presume that all candidate creep laws are approximated by a linear law in this range. Hence our estimate of $\zeta$ is probably correct. The other parts of Fig. 7 depict how various non-linear creep laws may affect our estimate of the absolute shear stress. Figure 7(b) shows the case where the estimate for stress is correct even though the creep law is arbitrarily complicated between a slip velocity of zero and $R$. Note that the steady state system path will never visit the complicated part of the curve at lower velocities. In contrast, Figs. 7(c) and (d) show cases where the post-seismic deformation sees a linear creep law with the same slope as in the purely linear case, yet use of that slope to infer the absolute stress level will yield values too high or low due to the large change in slope at slip velocities less than $R$. Since observed earthquake stress drops are similar to or larger than our $\sigma_{\text {creepAV }}$ estimates, the range of stress extrapolation is smaller than the "interrogated" stress range. But of course, it is possible that nature is using a deep creep constitutive law like those in either Figs. 7(c) or (d).

Is there any way to "interrogate" the creep law in the low slip velocity region? For the 2-D steady-state solutions, the answer is "no". Even if earthquakes randomly change their stress drop from one cycle to the next, the creep system will always be at velocities higher than about $s^{\prime}=(2 / 3) R$. Looking at the graphical $(u, v)$ space of Fig. 5 , we can only place the system closer to the $u^{\prime}=0$ line if some outside influence perturbs the system by a rapid decrease in $f_{2}$ or increase in $f_{1}$. This may be possible with a 3-D system where stress changes in adjacent subduction zone segments might temporarily increase the landward directed force on the creeper block, thus allowing it to temporarily slow down and thus interrogate the constitutive law between the origin and $s^{\prime}=(2 / 3) R$. I encourage the geodetic community to look for possible test cases of this scenario. Until then, the above values for $\sigma_{\text {creepAV }}$ may be our best estimates of the absolute shear stress acting on the deep creep interface of subduction zones.

\section{Implications and Conclusions}

The Savage model will continue to be the most effective model to relate geodetic information to plate interface slip in subduction zones. As shown in Fig. 8, the basic Savage model assigns a uniform creep velocity to the deep creep region, though Savage and others have allowed depth variations in the creep rate in some studies (e.g., Savage et al., 1998). To date, most of the improvement in subduction zone modeling has been to use a 3-D Savage model and data inversion for "backslip" in the seismogenic zone (e.g., Yabuki and Matsu'ura, 1992). Most current studies still assume uniform creep rate in the deep creep portion, and they assume that this rate is plate tectonic rate unless the data disagree with this assumption. The continued expansion of GPS networks will allow inversion for deep creep slip in future studies. In the meantime, I suggest that a simple formula can be used to prescribe the "backslip deficit" in the deep creep region over the earthquake cycle. Figure 8(b) shows this modification to the Savage model. The key idea is to use the simple formula for the ratio of deep creep rate to plate tectonic rate as a function of time through the earthquake cycle. As discussed above, the two-block model predicts that geodetic measurements would see an approximately linear strain rate in the coastal region during the inter-seismic phase, but at different rates as the $\left(t_{\text {fail }} / \tau\right)$ ratio varies from less than one to more than one. The case of $\left(t_{\text {fail }} / \tau\right) \ll 1$ is exactly the situation for the basic Savage model, where the deep creep slip velocity is nearly $R$ throughout the entire seismic cycle. But for the case of $\left(t_{\text {fail }} / \tau\right) \gg 1$, the behavior is quite different as there will be a distinct rapid post-seismic creep followed by a nearly constant creep rate on the deep creep zone through most of the earthquake cycle, but at a rate less than $R$. The formula that gives the creep rate throughout the seismic cycle depends on just two parameters; the $\left(t_{\text {fail }} / \tau\right)$ ratio and $\kappa_{2}$, which just depends on subduction zone geometry:

$$
s^{\prime}\left(t ; t_{\text {fail }} / \tau, \kappa_{2}\right)=R\left[1-\kappa_{2}(1-J \exp (t / \tau)] .\right.
$$

Geodetic observations from the Nankai, Alaska, and Chile subduction zones all show a rapid post-seismic deep creep with a characteristic time much less than the great earthquake recurrence time. If we generalize this result to all subduction zones, then the case of $\left(t_{\text {fail }} / \tau\right) \gg 1$ is the most prevalent case. In most other subduction zones, there are no geodetic observations of the rapid post-seismic deep creep, but there is still a clue to its existence in the inter-seismic phase. Recall that for $\left(t_{\text {fail }} / \tau\right) \gg 1$, the inter-seismic deep creep quickly obtains its asymptotic inter-seismic value of:

$$
s^{\prime}\left(t>\tau ; t_{\text {fail }} / \tau \gg 1, \kappa_{2}\right) \Rightarrow R\left(1-\kappa_{2}\right) .
$$

Thus, the prediction would be that geodetic measurements made in the inter-seismic phase at a "typical" subduction zone would be best matched by the modified Savage model with a deep creep velocity of $R\left(1-\kappa_{2}\right)$, not $R$. Recall that $\left(1-\kappa_{2}\right)$ has a value of about $(2 / 3)$ for $W_{1}=W_{2}$. It is somewhat counter-intuitive that only in the extreme case of high interface viscosity with creep stresses much higher than earthquake stress drops that would we expect to see the basic Savage model of Fig. 8(a).

There are some geodetic estimates of the deep creep slip velocity in a few subduction zones. Note that a geodetic survey that measures the absolute motion of a point above the locked seismogenic zone to a point in the plate interior (e.g., VLBI methods) should measure $R$; this measurement will not be sensitive to the deep creep velocity. One must use observations sensitive to the strain between the seismogenic and creep block. Recent results for the Nankai and Alaska appear to fit the coastal strain rate data with deep creep slip rates close to-or possibly even larger than-the plate tectonic rate of $R$ (see references for Nankai and Alaska). Thus, current geodetic results of the inter-seismic strain rate violate this prediction of the model. On the other hand, since large post-seismic creep was also observed at both Nankai and Alaska, it seems that the geodetic observations are not compatible with a steady-state system-independent of any model which seeks to make a quantitative connection (see Sato and Matsu'ura, 1992, for related discussion). I regard this post-seismic/inter-seismic mismatch as one of the more interesting mysteries about seismogenic/creep interaction during the earthquake cycle. 


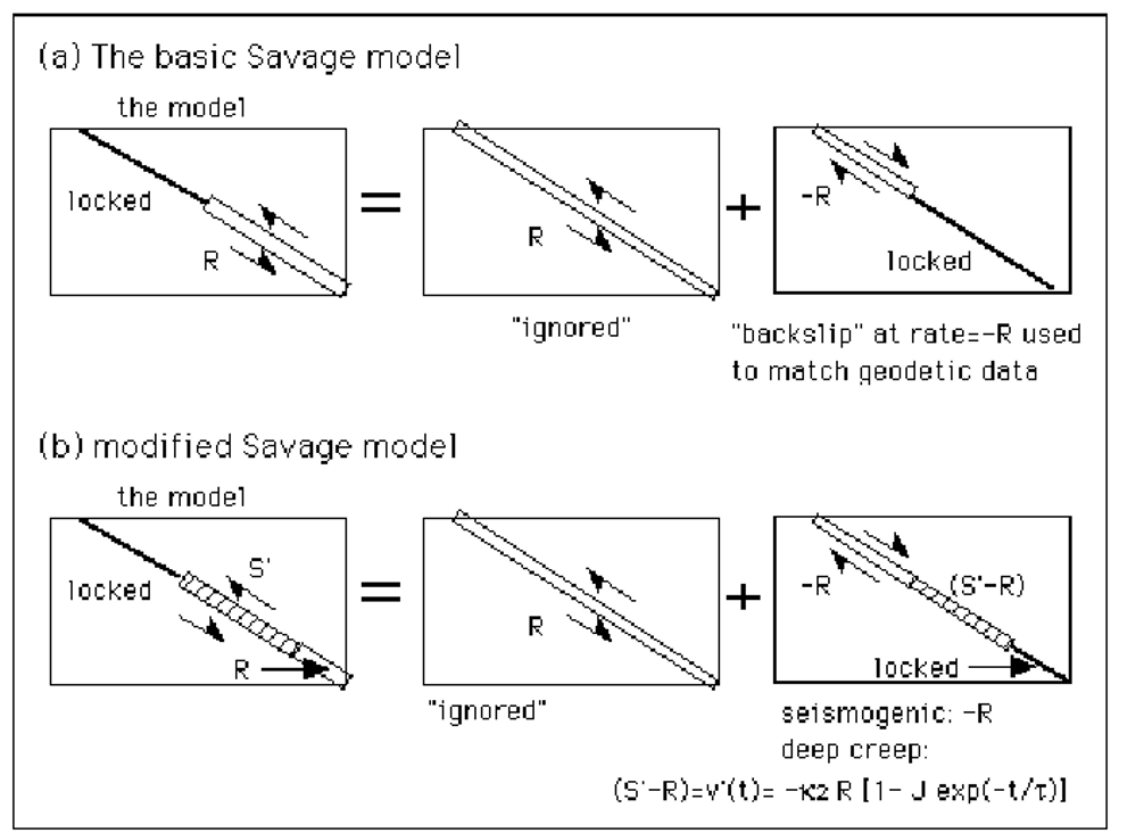

Fig. 8. The two-block model offers a formula for the slip rate in the deep creep plate interface. (a) shows the basic Savage model, where (b) shows the modified Savage model (e.g., Savage et al., 1998), with the formula from the two-block model. This formula contains both the post-seismic and inter-seismic phases of deep creep.

\subsection{Summary of model results}

The simple two-block model for seismogenic/deep creep zone interaction with stick-slip and linear creep interface behavior allows an analytic solution for the entire seismic cycle in subduction zones. Spatial and temporal integrals of the analytic solution are readily obtained, and the resultant algebraic relations are easily inverted so that several key model parameters can be estimated from observations. Aside from basic subduction zone geometry, the most important observation is $\tau$, the characteristic time for post-seismic deep creep. $\tau$ has been observed in several subduction zones. e.g., Nankai, Alaska, Chile, and possibly other zones (Kasahara, 1975; Heki et al., 1997), and it varies from 5 to 15 years. Estimates for $\tau$ allow us to calculate the deep creep interface viscosity, and then combined with another algebraic consequence of the simple model, we can calculate the average absolute level of shear stress acting on the deep creep interface. Values for the Nankai, Alaska, and Chile range from about 1 to 17 bars. To summarize how this procedure works, the previous great earthquake provides a "stress test" for the creep interface, and we extract the material property of interface viscosity from the observed $\tau$. Then, the average creep stress is simply the product of the interface viscosity and the plate tectonic rate. This last result, remarkable for its simplicity and ability to produce a value for absolute stress along the plate boundary, does assume that we can extrapolate the measured linear creep law back to zero velocity. This assumption should be vigorously tested by future studies.

All of the observed values for $\tau$ are much smaller than great earthquake recurrence times in these subduction zones. Hence the key system ratio of $\left(t_{\text {fail }} / \tau\right)$ is much larger than one, which then makes several predictions for deformation over the seismic cycle. The predictions for the
( $\left.\Delta u_{\text {post }} / \Delta u_{\text {coseismic }}\right)$ ratio have values close to $(1 / 3)$, observations of this ratio vary from less than $(1 / 10)$ to more than $(1 / 2)$. The inter-seismic creep zone slip rate is a more severe test for the model. For the case of $\left(t_{\text {fail }} / \tau\right) \gg 1$, the inter-seismic creep velocity across the deep creep interface must be less than $R$, and should be about $(2 / 3) R$ for the subduction zones considered here. More complicated theoretical models should produce this same conclusion, though the numerical value of the factor could be slightly different. Yet many geodetic studies in several subduction zones find that the strain data in the coastal region (i.e. above the seismogenic/creep interface) can be matched with deep creep velocities of $R$. Thus, there is a mismatch between the model prediction and the observed rates of deep creep. To construct a better model to match observed inter-seismic creep rates, one must violate one or more of the fundamental constraints. For example, you might argue that despite thousands of earthquake cycles in the Nankai, Alaska, and Chile subduction zones, these systems are far away from the steady-state path. Or, you could invent a stick-creep-stick constitutive law for the deep creep interface that would produce a creep rate of $R$ over a portion of the seismic cycle. To make progress in understanding the earthquake cycle and seismogenic/creep interaction, we need many more high-quality geodetic results that can point us in the right direction.

Acknowledgments. Thanks to the organizers of the Kochi meeting on Great Earthquake Recurrence and the Seismic Coupling workshop at GSJ in Tsukuba during January, 1999. These two meetings provided stimulating discussions on various issues of the earthquake cycle and earthquake occurrence in subduction zones. This research was partially supported by the National Science Foundation (EAR9725175 and OCE-9905503 to LJR). 


\section{References}

Ando, M., Source mechanism and tectonic significance of historical earthquakes along the Nankai Trough, Japan, Tectonophysics, 27, 119-140, 1975.

Ando, M., A fault model of the 1946 Nankaido earthquake derived from tsunami data, Phys. Earth Planet. Int., 28, 320-336, 1982.

Barrientos, S. E., G. Plafker, and E. Lorca, Postseismic coastal uplift in southern Chile, Geophys. Res. Lett., 19, 701-704, 1992.

Brown, L. D., R. Reilinger, S. Holdahl, and E. Balazs, Postseismic crustal uplift near Anchorage, Alaska, J. Geophys. Res., 82, 3369-3378, 1977.

Cifuentes, I. and P. Silver, Low-frequency source characteristics of the great 1960 Chilean earthquake, J. Geophys. Res., 94, 643-664, 1989.

Dieterich, J., Constitutive properties of faults with simulated gouge, in $\mathrm{Me}$ chanical Behavior of Crustal Rocks, AGU Geophys. Mono. 24, pp. 103 120, American Geophysical Union, Washington, D.C., 1981.

Dixon, T. H., GPS measurement of relative motion of the Cocos and Caribbean plates and strain accumulation across the middle America trench, Geophys. Res. Lett., 20, 2167-2170, 1993.

Dmowska, R., G. Zheng, and J. Rice, Seismicity and deformation at convergent margins due to heterogeneous coupling, J. Geophys. Res., 101, 3015-3030, 1996.

Heki, K., S. Miyazaki, and H. Tsuji, Silent fault slip following an interplate thrust earthquake at the Japan trench, Nature, 386, 595-598, 1997.

Hirahara, K., FEM modeling for seismic cycle of great interplate earthquakes following the rate- and state-dependent friction law-preliminary analysis, in Recurrence of great interplate earthquakes and its mechanism (Kochi Workshop proceedings), pp. 181-186, Sci. \& Tech. Agency, Kochi, 1999.

Hyndman, R., K. Wang, and M. Yamano, Thermal constraints on the seismogenic portion of the southwestern Japan subduction thrust, J. Geophys. Res., 100, 15,373-15,392, 1995.

Ito, T., S. Yoshioka, and S. Miyazaki, Interplate coupling in southwest Japan deduced from inversion analysis of GPS data, Phys. Earth Planet. Int., 1999 (submitted).

Kanamori, H. and D. L. Anderson, Theoretical basis of some empirical relations in seismology, Bull. Seism. Soc. Am., 65, 1073-1095, 1975.

Kanamori, H. and J. Cipar, Focal process of the great Chilean earthquake May 22, 1960, Phys. Earth Planet. Int., 9, 128-136, 1974.

Kasahara, K., Aseismic faulting following the 1973 Nemuro-Oki earthquake, Hokkaido, Japan (a possibility), Pageoph, 113, 127-139, 1975.

Mogi, K., Temporal variation of crustal deformation during the days preceding a thrust-type great earthquake - the 1944 Tonankai earthquake of magnitude 81, Pageoph, 122, 765-780, 1985.

Nomanbhoy, N. and L. J. Ruff, A simple discrete elements model of large multiplet earthquakes, J. Geophys. Res., 101, 5707-5724, 1996.

Peacock, S. M., Thermal and petrologic structure of subduction zones, in Subduction: Top to Bottom, edited by G. Bebout, D. Scholl, S. Kirby, and J. Platt, pp. 119-133, AGU Geophys. Mono. 96, American Geophys Union, Washington, D.C., 1996.

Plafker, G., Alaskan earthquake of 1964 and Chilean earthquake of 1960 , implications for arc tectonics, J. Geophys. Res., 77, 901-925, 1972.

Plafker, G. and J. C. Savage, Mechanism of the Chilean earthquakes of May 21 and May 22, 1960, Geol. Soc. Am. Bull., 81, 1001-1030, 1970.

Rice, J. R. and S. T. Tse, Dynamic motion of a single degree of freedom system following a rate and state dependent friction law, J. Geophys. Res., 91, 521-530, 1986.

Ruff, L. J., Asperity distributions and large earthquake occurrence in sub- duction zones, Tectonophys., 211, 1-23, 1992.

Ruff, L. J., Dynamic stress drop of recent earthquakes: variations within subduction zones, Pageoph, 154, 409-431, 1999.

Sagiya, T., Spatio-temporal variation of interplate coupling along the Nankai Trough deduced from geodetic data inversion, in Recurrence of great interplate earthquakes and its mechanism (Kochi Workshop proceedings), pp. 148-152, Sci. \& Tech. Agency, Kochi, 1999.

Sagiya, T. and W. Thatcher, Coseismic slip resolution along a plate boundary megathrust: the Nankai trough, southwest Japan, J. Geophys. Res., 104, 1111-1129, 1999

Sato, T. and M. Matsu'ura, Cyclic crustal movement, steady uplift of marine terraces, and evolution of the island arc-trench system in southwest Japan, Geophys. J. Int., 111, 617-629, 1992.

Savage, J. C., Interseismic uplift at the Nankai subduction zone, southwest Japan, 1951-1990, J. Geophys. Res., 100, 6339-6350, 1995.

Savage, J. C. and W. Thatcher, Interseismic deformation at the Nankai Trough, Japan, subduction zone, J. Geophys. Res., 97, 11,117-11,135, 1992.

Savage, J. C., J. Svarc, W. Prescott, and W. Gross, Deformation across the rupture zone of the 1964 Alaska earthquake, 1993-1997, J. Geophys. Res., 103, 21,275-21,283, 1998 .

Scholz, C. H., The Mechanics of Earthquakes and Faulting, 439 pp., Cambridge Univ. Press, Cambridge, 1990.

Seno, T., S. Stein, and A. Gripp, A model for the motion of the Philippine Sea plate consistent with NUVEL-1 and geological data, J. Geophys. Res., 98, 17,941-17,948, 1993.

Stuart, W. D., Forecast model for great earthquakes at the Nankai Trough subduction zone, Pageoph, 126, 619-642, 1988.

Stuart, W. and T. Sagiya, Initial results from a three-dimensional fault model for earthquake cycles at the Nankai subduction zone, in Recurrence of great interplate earthquakes and its mechanism (Kochi Workshop proceedings), pp. 173-180, Sci. \& Tech. Agency, Kochi, 1999.

Tabei, T., Lateral heterogeneity of subduction process at the Nankai Trough inferred from GPS data, in Recurrence of great interplate earthquakes and its mechanism (Kochi Workshop proceedings), pp. 6-12, Sci. \& Tech. Agency, Kochi, 1999.

Tanioka, Y. and K. Satake, Coseismic slip distribution of the 1946 Nankai earthquake and aseismic slips caused by the earthquake, Earth Planets Space, 53, this issue, 235-241, 2001.

Thatcher, W. and J. Rundle, A visco-elastic coupling model for cyclic deformation due to periodically repeated earthquakes at subduction zones, J. Geophys. Res., 89, 7631-7640, 1984.

Tichelaar, B. W. and L. J. Ruff, Depth of seismic coupling along subduction zones, J. Geophys. Res., 98, 2017-2037, 1993.

Wang, K. and J. He, Mechanics of low-stress forearcs: Nankai and Cascadia, J. Geophys. Res., 104, 15,191-15,205, 1999.

Yabuki, T. and M. Matsu'ura, Geodetic data inversion using a Bayesian information criterion for spatial distribution of fault slip, Geophys. J. Int., 109, 363-375, 1992.

Yoshioka, S., Three-dimensional numerical simulation of displacement and strain fields associated with the 1944 Tonankai and 1946 Nankai earthquakes, in Recurrence of great interplate earthquakes and its mechanism (Kochi Workshop proceedings), pp. 203-209, Sci.\& Tech. Agency, Kochi, 1999.

L. J. Ruff (e-mail: ruff@geo.lsa.umich.edu) 